\title{
Water requirements for major crops in different agro-climatic zones of Iraqi Kurdistan using by CROPWAT 8.0
}

\author{
Dawod Rasooli Keya \\ (Horticulture, Khabat ATI/ Erbil Polytechnic University, Iraqi Kurdistan)
}

\begin{abstract}
The study was performed by using FAO Penman-Monteith equation. Required parameters were set for 9 synoptic stations in Iraqi Kurdistan for a period from 2004 to 2012. Sangasar and Karkuk were selected as two different agro-climatic zones. Data were processed by (CROPWAT 8.0) program, and the values of ETo and effective rainfall were calculated. Several crops were selected in the study including alfalfa, barley, beans, cabbage, grapes, maize, potatoes, sunflower, melons, tomatoes and wheat. Crop coefficients (Kc) were taken from tables of FAO under medium soil conditions. The results show that potential evapotranspiration in Sangasar zone was $0.9-8 \mathrm{~mm} /$ day, whereas in Karkuk zone was 1.5 to $12 \mathrm{~mm} /$ day. The highest water requirement was estimated for grape in the Sangasar zone (Soran) $987.6 \mathrm{~mm} /$ day and $1635.1 \mathrm{~mm} /$ day for Karkuk zone (Makhmur). In the same way, the minimum water requirement was estimated for alfalfa (first mowing) in Sangasar zone (Penjwen) 224.1mm/day and $269.7 \mathrm{~mm} /$ day for Karkuk zone (Chamchamal).
\end{abstract}

Keywords - potential evapotranspiration, crop water requirement, Penman-Monteith method, CROPWAT, Iraqi Kurdistan

\section{INTRODUCTION}

Water resources management has been a challenge in Iraqi Kurdistan due to precipitation shortage in recent years. Economically crops production has direct relationship with irrigation. However, irrigation scheduling has been based on the predicted crop water requirements (CWR), which is the total water needed for evapotranspiration from planting to harvest for a given crop in a specific climate regime. When adequate soil water is maintained by rainfall and/or irrigation, it does not limit plant growth and crop yield (Hess, 2005). CWR is calculated for a given crop under the different climatic conditions. (FAO, 2005) defined that CWR is the depth (or amount) of water needed to meet the water loss through evapotranspiration. In other words, it is the amount of needed water by the various crops to grow optimally. The crop water requirement always refers to a crop grown under optimal conditions, i.e. a uniform crop, actively growing, completely shading the ground, free of diseases, and favorable soil conditions (including fertility and water). The CWR mainly depends on the climate, the crop type and the growth stage of the crops. The effects of various climates on evapotranspiration are incorporated into reference evapotranspiration $\left(\mathrm{ET}_{\mathrm{o}}\right)$. In this study, $\mathrm{ET}_{\mathrm{o}}$ is calculated by using the FAO PenmanMonteith method (FAO, 1998):

$$
\lambda \mathrm{ET}=\frac{\Delta\left(\mathrm{R}_{\mathrm{n}}-\mathrm{G}\right)+\rho_{\mathrm{a}} \mathrm{c}_{\mathrm{p}} \frac{\left(\mathrm{e}_{\mathrm{s}}-\mathrm{e}_{\mathrm{a}}\right)}{\mathrm{r}_{\mathrm{a}}}}{\Delta+\gamma\left(1+\frac{\mathrm{r}_{\mathrm{s}}}{\mathrm{r}_{\mathrm{a}}}\right)}
$$

Where $R_{n}$ is the net radiation, $G$ is the soil heat flux, $\left(e_{s}-e_{a}\right)$ represents the vapour pressure deficit of the air, $\rho_{\mathrm{a}}$ is the mean air density at constant pressure, $c_{\mathrm{p}}$ is the specific heat of the air, $\Delta$ represents the slope of the saturation vapour pressure temperature relationship, $\gamma$ is the psychrometric constant, and $r_{s}$ and $r a$ are the (bulk) surface and aerodynamic resistances.

CWR or $\mathrm{ET}_{\mathrm{c}}$ is calculated by multiplying the reference crop evapotranspiration, $\mathrm{ET}_{\mathrm{o}}$ and a crop coefficient, Kc values. The effects of characteristics that distinguish field crops from grass are integrated into the crop coefficient. In order to, tabulated Kc values are used for initial, development, mid and late stages of crop growing (FAO, 1977). 


\section{MATERIALS AND METHODS}

Iraqi Kurdistan region is located at latitude 35.00 to 37.30 , longitude 42.20 to 46.20 and includes three governorates which are Erbil, Dohuk and Sulaymaniyah (Figure 1). Kurdistan region has been divided into Sangasar and Karkuk agro-climatic zones (FAO/UNESCO/WMO, 1962). Sangasar zone consists of hilly areas and high mountains with 600 to $1000 \mathrm{~mm}$ of rainfall per annum. The vegetation is mainly oak forest on slopes between 500 and 2000 meters altitude; whereas mostly grasses between 2000 to 4000 meters and alpine plants above 4000 meters. Karkuk zone includes the main dry farming area of the region with 200 to $600 \mathrm{~mm}$ rainfall. The summer is hot and dry but the winter is cold and rainy.

Estimation of $\boldsymbol{E T}_{\boldsymbol{o}}$ : (FAO, 1992), (Smith et al., 1991) and (Smith, 1992) reported that CROPWAT is a practical tool (software) used to help agrometeorologists, agronomists and irrigation engineers to carry out standard calculations for evapotranspiration and crop water use studies, and

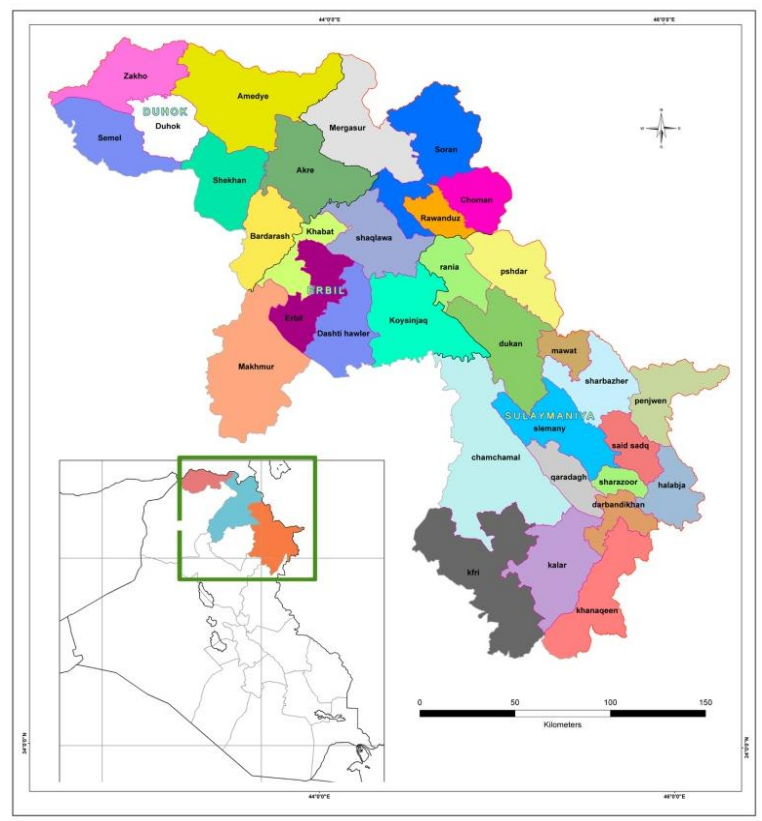

Figure 1. Map of the governorates of Kurdistan region more accurate design and management for irrigation schemes.

In order to, CROPWAT 8.0 was used to study of 9 meteorological stations. The respective climatic data were collected from the agro-meteorological department /Kurdistan ministry of agriculture and water resources and arranged by using Excel program. Table 1 shows summary statistics of meteorological stations.

Table1. Main meteorological data recorded in study areas (2004-2012)

\begin{tabular}{|c|c|c|c|c|c|c|c|c|c|c|}
\hline \multirow{3}{*}{ Station } & \multirow{3}{*}{ zone } & \multirow[b]{3}{*}{ Lat. } & \multirow[b]{3}{*}{ Log. } & \multirow[b]{3}{*}{ Alt., m } & \multicolumn{5}{|c|}{ Annual average } & \multirow{3}{*}{$\begin{array}{c}\text { Annual } \\
\text { Rainfall, mm }\end{array}$} \\
\hline & & & & & \multicolumn{2}{|c|}{ Air temp. ${ }^{\circ} \mathrm{C}$} & \multirow{2}{*}{ Humidity, \% } & \multirow{2}{*}{$\begin{array}{l}\text { Wind } \\
\text { Speed, m/s }\end{array}$} & \multirow{2}{*}{$\begin{array}{l}\text { Sunshine } \\
\text { duration, h }\end{array}$} & \\
\hline & & & & & Min. & Max. & & & & \\
\hline Akre & Sangasar & $36^{\circ} 50^{\prime} \mathrm{N}$ & $43^{\circ} 51^{\prime} \mathrm{E}$ & 636 & 14.6 & 23.2 & 42.0 & 1.6 & 7.6 & 477.7 \\
\hline Chamchamal & Karkuk & $35^{\circ} 33^{\prime} \mathrm{N}$ & $45^{\circ} 50^{\prime} \mathrm{E}$ & 708 & 14.2 & 26.4 & 41.0 & 0.8 & 6.9 & 387.5 \\
\hline Duhok & Sangasar & $36^{\circ} 50^{\prime} \mathrm{N}$ & $43^{\circ} 00^{\prime} \mathrm{E}$ & 569 & 15.0 & 26.2 & 44.9 & 1.1 & 7.6 & 488.5 \\
\hline Erbil & Karkuk & $36^{\circ} 12^{\prime} \mathrm{N}$ & $44^{\circ} 02^{\prime} \mathrm{E}$ & 420 & 15.7 & 27.4 & 47.4 & 2.3 & 7.9 & 395.7 \\
\hline Makhmur & Karkuk & $35^{\circ} 46^{\prime} \mathrm{N}$ & $43^{\circ} 35^{\prime} \mathrm{E}$ & 270 & 16.6 & 29.4 & 42.7 & 3.7 & 7.9 & 242.5 \\
\hline Penjwen & Sangasar & $35^{\circ} 37^{\prime} \mathrm{N}$ & $45^{\circ} 56^{\prime} \mathrm{E}$ & 1302 & 7.6 & 17.8 & 59.9 & 3.8 & 7.4 & 1002.9 \\
\hline Soran & Sangasar & $36^{\circ} 39^{\prime} \mathrm{N}$ & $44^{\circ} 32^{\prime} \mathrm{E}$ & 680 & 12.2 & 24.3 & 62.0 & 2.0 & 7.6 & 653.1 \\
\hline Sulaymaniyah & Sangasar & $35^{\circ} 33^{\prime} \mathrm{N}$ & $45^{\circ} 27^{\prime} \mathrm{E}$ & 885 & 14.6 & 25.4 & 45.8 & 1.3 & 7.3 & 622.5 \\
\hline Zakho & Sangasar & $37^{\circ} 80^{\prime} \mathrm{N}$ & $42^{\circ} 41^{\prime} \mathrm{E}$ & 444 & 14.7 & 26.7 & 38.6 & 1.1 & 6.8 & 528.6 \\
\hline
\end{tabular}

Loamy soil was considered as a case study. Total available soil moisture (FC-WP) for this type of soil was equal to $180 \mathrm{~mm} / \mathrm{meter}$ and maximum rain infiltration rate was equal to $30 \mathrm{~mm} /$ day.

\section{RESULTS AND DISCUSSION}

Annual potential evapotranspiration $\left(\mathrm{ET}_{\mathrm{o}}\right)$ had estimate values between 1339 - $2382 \mathrm{~mm}$ for Zakho and Makhmur respectively. The highest and lowest values of $\mathrm{ET}_{\mathrm{o}}$ were recorded in July and January which were 12 $\mathrm{mm} /$ day and $0.9 \mathrm{~mm} /$ day for Makhmur and Soran, respectively (Table 2).

Table 2: Estimated monthly and annual ETo for studied stations

\begin{tabular}{|c|c|c|c|c|c|c|c|c|c|c|c|c|c|c|}
\hline \multirow{2}{*}{ Station } & \multirow{2}{*}{ Zone } & \multicolumn{12}{|c|}{ ETo, mm/day } & \multirow{2}{*}{$\begin{array}{l}\text { Annual } \\
\mathrm{mm}\end{array}$} \\
\hline & & Jan. & Feb. & Mar. & Apr. & may & Jun. & Jul. & Aug. & Sep. & Oct. & Nov. & Dec. & \\
\hline Akre & Sangasar & 1.0 & 1.5 & 2.2 & 3.6 & 5.8 & 6.7 & 7.0 & 7.1 & 5.6 & 3.8 & 2.0 & 1.3 & 1451 \\
\hline Chamchamal & Karkuk & 1.7 & 2.3 & 3.0 & 4.0 & 4.7 & 5.3 & 5.0 & 6.6 & 5.0 & 3.3 & 2.4 & 1.7 & 1361 \\
\hline Duhok & Sangasar & 1.2 & 1.5 & 2.6 & 3.4 & 5.1 & 6.4 & 6.7 & 6.2 & 4.9 & 3.3 & 1.8 & 1.3 & 1352 \\
\hline Erbil & Karkuk & 1.5 & 1.9 & 3.1 & 4.3 & 6.6 & 8.3 & 8.5 & 8.1 & 6.3 & 4.5 & 2.4 & 1.6 & 1738 \\
\hline
\end{tabular}




\begin{tabular}{lllllllllllllll}
\hline Makhmur & Karkuk & 1.9 & 2.5 & 3.9 & 5.8 & 8.7 & 11.5 & 12.0 & 11.4 & 9.0 & 6.1 & 3.3 & 2.1 & 2382 \\
\hline Penjwen & Sangasar & 0.9 & 1.2 & 2.1 & 3.1 & 4.5 & 7.2 & 7.7 & 7.9 & 6.6 & 3.6 & 1.7 & 1.4 & 1458 \\
\hline Soran & Sangasar & 0.9 & 1.2 & 2.5 & 3.3 & 5.2 & 7.5 & 8.0 & 7.7 & 6.0 & 3.3 & 1.8 & 1.1 & 1479 \\
\hline Sulaymaniyahh & Sangasar & 1.1 & 1.6 & 2.6 & 3.4 & 4.5 & 6.8 & 7.1 & 6.8 & 5.1 & 3.3 & 1.8 & 1.3 & 1385 \\
\hline Zakho & Sangasar & 1.2 & 1.5 & 2.5 & 3.4 & 5.1 & 6.4 & 6.7 & 6.2 & 4.9 & 3.2 & 1.8 & 1.2 & 1339 \\
\hline
\end{tabular}

Crop water requirements $($ CWR) and irrigation requirement (IR): The CWR module includes calculations, producing the irrigation water requirement of the crop on a monthly basis and total growing season. Irrigation requirement is different between the crop evapotranspiration under standard conditions $\left(\mathrm{ET}_{\mathrm{c}}\right)$ and the effective rainfall. A summary of the results, which explains the different CWR and IR calculation for selected crops, is showed in the tables 3 to 13 .

Alfalfa

The highest CWR by alfalfa is $428.5 \mathrm{~mm}$ at Makhmur, and the lowest at Penjwen is $224.1 \mathrm{~mm}$; whereas, the highest IR by alfalfa is $361.4 \mathrm{~mm}$ at Makhmur and the lowest at Penjwen is $56.1 \mathrm{~mm}$ (Table 3 ).

Table 3: Estimated CWR and IR for alfalfa (first cutting) in the studied stations

\begin{tabular}{lcccccccc}
\hline \multirow{2}{*}{ Station } & \multicolumn{3}{c}{ Mar. } & \multicolumn{2}{c}{ Apr. } & \multicolumn{2}{c}{ May } & \multicolumn{2}{c}{ Total } \\
\cline { 2 - 9 } & CWR & IR & CWR & IR & CWR & IR & CWR & IR \\
\hline Akre & 43.5 & 8.4 & 124 & 68.5 & 85 & 74.1 & 252.5 & 151.0 \\
\hline Chamchamal & 55.9 & 21.2 & 138.6 & 78.9 & 75.2 & 66.2 & 269.7 & 166.3 \\
\hline Duhok & 47.6 & 13.8 & 118 & 66.8 & 74.9 & 62.5 & 240.5 & 143.1 \\
\hline Erbil & 59.7 & 14.8 & 154.6 & 113.5 & 100.7 & 92.2 & 315.0 & 220.5 \\
\hline Makhmur & 78.4 & 54.4 & 213 & 176 & 137.1 & 131 & 428.5 & 361.4 \\
\hline Penjwen & 41.1 & 0 & 113.5 & 13.6 & 69.5 & 42.5 & 224.1 & 56.1 \\
\hline Soran & 46.6 & 0 & 116.8 & 30.7 & 77.9 & 62 & 241.3 & 92.7 \\
\hline Sulaymaniyah & 48.3 & 0 & 117 & 36 & 66.5 & 43.2 & 231.8 & 79.2 \\
\hline Zakho & 46.9 & 6.3 & 117 & 68.6 & 74.6 & 63.6 & 238.5 & 138.5 \\
\hline
\end{tabular}

\section{Barley}

The highest CWR by barley is $702.8 \mathrm{~mm}$ at Makhmur and the lowest at Penjwen $368.4 \mathrm{~mm}$; while, the highest IR by barley is $496.1 \mathrm{~mm}$ at Makhmur and the lowest at Penjwen $49.8 \mathrm{~mm}$ (Table 4).

Table 4: Estimated CWR and IR for barley in the studied stations

\begin{tabular}{|c|c|c|c|c|c|c|c|c|c|c|c|c|c|c|c|c|}
\hline \multirow{2}{*}{ Station } & \multicolumn{2}{|c|}{ Nov. } & \multicolumn{2}{|c|}{ Des. } & \multicolumn{2}{|c|}{ Jan. } & \multicolumn{2}{|c|}{ Feb. } & \multicolumn{2}{|c|}{ Mar. } & \multicolumn{2}{|c|}{ Apr. } & \multicolumn{2}{|c|}{ May } & \multicolumn{2}{|c|}{ Total } \\
\hline & CWR & IR & CWR & IR & CWR & IR & CWR & IR & CWR & IR & CWR & IR & CWR & IR & CWR & IR \\
\hline Akre & 16.9 & 0 & 24.8 & 0 & 25.8 & 0 & 41 & 0 & 77.2 & 31.2 & 118.6 & 63.2 & 96.9 & 81.2 & 401.2 & 175.6 \\
\hline Chamchamal & 21.9 & 6 & 33.1 & 4.3 & 29.6 & 0 & 46.6 & 0 & 104.8 & 64.3 & 136.6 & 76.9 & 85.1 & 73.7 & 457.7 & 225.2 \\
\hline Duhok & 16.3 & 0 & 24.3 & 0 & 29.2 & 0 & 44.8 & 0 & 86.8 & 45 & 114.8 & 63.7 & 86.4 & 67.1 & 402.6 & 175.8 \\
\hline Erbil & 20.2 & 1 & 30.6 & 0 & 36.4 & 0 & 57.2 & 1.3 & 111.1 & 55.7 & 150.7 & 109.5 & 115.6 & 102.9 & 521.8 & 270.4 \\
\hline Makhmur & 27.7 & 16.6 & 39.7 & 14.3 & 48.2 & 6.7 & 73.8 & 14.2 & 148 & 124 & 208.4 & 171.3 & 157 & 149 & 702.8 & 496.1 \\
\hline Penjwen & 15 & 0 & 25 & 0 & 23.4 & 0 & 36 & 0 & 77 & 0 & 111.3 & 11.5 & 80.7 & 38.3 & 368.4 & 49.8 \\
\hline Soran & 15.6 & 0 & 20.8 & 0 & 23.4 & 0 & 36.2 & 0 & 86.4 & 8.3 & 113.8 & 27.7 & 90.6 & 67.9 & 386.8 & 103.9 \\
\hline Sulayma & 15.4 & 0 & 24.5 & 0 & 28 & 0 & 46.1 & 0 & 88.6 & 13.8 & 114.2 & 33.3 & 77.7 & 40 & 394.5 & 87.1 \\
\hline Zakho & 15.7 & 0 & 23.4 & 0 & 28.2 & 0 & 43.8 & 0 & 85.6 & 24.1 & 113.8 & 65.6 & 86 & 69.4 & 396.5 & 159.1 \\
\hline
\end{tabular}

\section{Beans}

The highest CWR by beans is $657.7 \mathrm{~mm}$ at Makhmur and the lowest at Penjwen $344.9 \mathrm{~mm}$; while, the highest IR by beans is $451.1 \mathrm{~mm}$ at Makhmur and the lowest at Penjwen is $34 \mathrm{~mm}$ (Table 5).

Table 5: Estimated CWR and IR for beans in the studied stations

\begin{tabular}{lccccccccccccccccc}
\hline \multirow{2}{*}{ Station } & \multicolumn{2}{c}{ Nov. } & \multicolumn{2}{c}{ Des. } & \multicolumn{2}{c}{ Jan. } & \multicolumn{2}{c}{ Feb. } & \multicolumn{2}{c}{ Mar. } & \multicolumn{2}{c}{ Apr. } & \multicolumn{2}{c}{ May } & \multicolumn{2}{c}{ Total } \\
\cline { 2 - 6 } & CWR & IR & CWR & IR & CWR & IR & CWR & IR & CWR & IR & CWR & IR & CWR & IR & CWR & IR \\
\hline Akre & 11.3 & 0 & 19.8 & 0 & 27.9 & 0 & 44.4 & 0 & 78.1 & 32 & 109.6 & 54.2 & 89.8 & 74.1 & 380.9 & 160.3 \\
\hline Chamchamal & 14.6 & 0.5 & 27 & 0 & 32.2 & 0 & 49.8 & 0 & 103.6 & 63.1 & 124.1 & 64.3 & 76.4 & 64.9 & 427.7 & 192.8 \\
\hline Duhok & 10.9 & 0 & 19.7 & 0 & 31.8 & 0 & 48.7 & 0 & 87.7 & 45.9 & 106.3 & 55.2 & 79.8 & 60.4 & 384.9 & 161.5 \\
\hline Erbil & 13.5 & 0 & 24.7 & 0 & 39.8 & 0 & 61.5 & 3.6 & 110.5 & 55.1 & 137.2 & 95.9 & 104.9 & 92.1 & 492.1 & 246.7 \\
\hline Makhmur & 18.4 & 7.4 & 32.5 & 7 & 53 & 11.5 & 78.9 & 19.4 & 146 & 122 & 188 & 151 & 140.9 & 132.8 & 657.7 & 451.1 \\
\hline Penjwen & 10 & 0 & 20.2 & 0 & 25.6 & 0 & 38.6 & 0 & 76.4 & 0 & 101 & 3.4 & 73.1 & 30.6 & 344.9 & 34 \\
\hline
\end{tabular}


Water requirements for major crops in different agro-climatic zones of Iraqi Kurdistan using by CROPWAT 8.0

\begin{tabular}{lcccccccccccccccc} 
Soran & 10.3 & 0 & 16.8 & 0 & 25.5 & 0 & 38.8 & 0 & 85.9 & 8 & 103.6 & 17.5 & 82.5 & 59.9 & 363.4 & 85.4 \\
\hline Sulaymaniyah & 10.2 & 0 & 19.7 & 0 & 30.6 & 0 & 49.9 & 0 & 89.3 & 14.3 & 105.8 & 24.8 & 71.5 & 34 & 377 & 73.1 \\
\hline Zakho & 10.5 & 0 & 18.8 & 0 & 30.6 & 0 & 47.3 & 0 & 86.4 & 24.9 & 105.4 & 57.2 & 79.4 & 62.8 & 378.4 & 144.9 \\
\hline
\end{tabular}

\section{Cabbage}

The highest CWR by cabbage is $844.7 \mathrm{~mm}$ at Makhmur and the lowest at Zakho 452.1mm; while, the highest IR by cabbage is $748.4 \mathrm{~mm}$ at Makhmur and the lowest at Zakho $283.9 \mathrm{~mm}$ (Table 6).

Table 6: Estimated CWR and IR for cabbage in the studied stations

\begin{tabular}{|c|c|c|c|c|c|c|c|c|c|c|c|c|c|c|}
\hline \multirow{2}{*}{ Station } & \multicolumn{2}{|c|}{ Aug. } & \multicolumn{2}{|c|}{ Sep. } & \multicolumn{2}{|c|}{ Oct. } & \multicolumn{2}{|c|}{ Nov. } & \multicolumn{2}{|c|}{ Des. } & \multicolumn{2}{|c|}{ Jan. } & \multicolumn{2}{|c|}{ Total } \\
\hline & CWR & IR & CWR & IR & CWR & IR & CWR & IR & CWR & IR & CWR & IR & CWR & IR \\
\hline Akre & 150.7 & 150.5 & 122.3 & 120.2 & 103.9 & 77.6 & 61.2 & 17.9 & 41.5 & 0 & 28 & 0 & 507.6 & 366.2 \\
\hline Chamchamal & 141.2 & 141.1 & 110.4 & 105.4 & 95.5 & 74.1 & 77.6 & 47 & 59 & 26.9 & 33 & 0 & 516.7 & 394.5 \\
\hline Duhok & 132.7 & 132.6 & 107.9 & 106.3 & 89.6 & 64.2 & 57.5 & 10.6 & 40.8 & 0 & 31.8 & 0 & 460.3 & 313.7 \\
\hline Erbil & 173.9 & 173.6 & 140.2 & 137.6 & 123.6 & 105.2 & 74.4 & 32.3 & 52.2 & 0 & 39.8 & 0 & 604.1 & 448.7 \\
\hline Makhmur & 245.2 & 245.1 & 198.5 & 195.1 & 172.4 & 165 & 104.8 & 84.7 & 70.1 & 44.7 & 53.7 & 13.8 & 844.7 & 748.4 \\
\hline Penjwen & 169.2 & 169 & 146.2 & 143.8 & 101.9 & 49.3 & 57.1 & 1.5 & 43.9 & 0 & 26.3 & 0 & 544.6 & 363.6 \\
\hline Soran & 165.2 & 164.5 & 132.5 & 128.6 & 92 & 45.6 & 56.8 & 9.4 & 35.5 & 0 & 25.5 & 0 & 507.5 & 348.1 \\
\hline Sulaymaniyah & 145.3 & 145.2 & 112.9 & 111 & 91.4 & 44.1 & 54.8 & 4.6 & 41.1 & 0 & 30.3 & 0 & 475.8 & 304.9 \\
\hline Zakho & 132 & 131.4 & 106.6 & 102.8 & 87.8 & 41.4 & 55.7 & 8.3 & 39.3 & 0 & 30.7 & 0 & 452.1 & 283.9 \\
\hline
\end{tabular}

\section{Grape}

The highest CWR by grape is $1635.1 \mathrm{~mm}$ at Makhmur and the lowest at Chamchamal 798.5mm; while, the highest total IR by grape is $1553.7 \mathrm{~mm}$ at Makhmur and the lowest at Chamchamal $682 \mathrm{~mm}$ (Table 7).

Table 7: Estimated CWR and IR for grape in the studied stations

\begin{tabular}{|c|c|c|c|c|c|c|c|c|c|c|c|c|c|c|c|c|c|c|}
\hline \multirow{2}{*}{ Station } & \multicolumn{2}{|c|}{ Feb. } & \multicolumn{2}{|c|}{ Mar. } & \multicolumn{2}{|c|}{ Apr. } & \multicolumn{2}{|c|}{ may } & \multicolumn{2}{|c|}{ Jun. } & \multicolumn{2}{|c|}{ Jul. } & \multicolumn{2}{|c|}{ Aug. } & \multicolumn{2}{|c|}{ Sep. } & \multicolumn{2}{|c|}{ Total } \\
\hline & CWR & IR & CWR & IR & CWR & IR & CWR & IR & CWR & IR & CWR & IR & CWR & IR & CWR & IR & CWR & IR \\
\hline Akre & 6.7 & 0 & 32.9 & 1.9 & 96 & 40.6 & 177.6 & 161.9 & 198.9 & 198.8 & 200.3 & 200.3 & 141.5 & 141.4 & 20.5 & 20.5 & 874.4 & 765.4 \\
\hline Chamchamal & 10 & 0 & 44.2 & 10.8 & 107.7 & 47.9 & 153.7 & 142.1 & 164.4 & 163.6 & 153.3 & 153.3 & 144.3 & 144.2 & 20.9 & 20.1 & 798.5 & 682 \\
\hline Duhok & 7.3 & 0 & 36.4 & 4.9 & 92.6 & 41.5 & 159.8 & 140.5 & 194.6 & 193.7 & 194.3 & 194.2 & 127.6 & 127.4 & 18.5 & 18.5 & 831.1 & 720.7 \\
\hline Erbil & 8.9 & 0 & 45.7 & 6.2 & 121.3 & 80.1 & 216.1 & 203.4 & 262 & 260.2 & 257.7 & 257.4 & 177.8 & 177.4 & 25.9 & 25.8 & 1115.4 & 1010.5 \\
\hline Makhmur & 11.1 & 0 & 60.5 & 36.5 & 170.6 & 133.5 & 302.8 & 294.8 & 384.4 & 384.1 & 390.3 & 390.2 & 274 & 273.9 & 41.4 & 40.7 & 1635.1 & 1553.7 \\
\hline Penjwen & 5.6 & 0 & 31.8 & 0 & 91.1 & 8.8 & 157.4 & 115 & 234.3 & 232.6 & 243.2 & 241.1 & 183.5 & 183.4 & 29.3 & 29.3 & 976.2 & 810.2 \\
\hline Soran & 5.9 & 0 & 35.9 & 0 & 92.7 & 16.8 & 173.3 & 150.6 & 238.1 & 236.9 & 244.5 & 243.9 & 171.5 & 171 & 25.7 & 25.7 & 987.6 & 844.9 \\
\hline Sulaymaniyah & 7.5 & 0 & 36.9 & 0 & 91 & 14.9 & 144 & 106.5 & 205.5 & 204.3 & 204.7 & 204.6 & 140.1 & 140 & 19.8 & 19.8 & 849.5 & 690.1 \\
\hline Zakho & 7.1 & 0 & 35.9 & 0 & 91.8 & 43.6 & 159.1 & 142.6 & 194.3 & 192.7 & 193.9 & 193.8 & 127 & 126.7 & 18.4 & 18.3 & 827.5 & 717.7 \\
\hline
\end{tabular}

Maize

The highest total CWR by maize is $1404.4 \mathrm{~mm}$ at Makhmur and the lowest at Chamchamal 635.8mm; while, the highest IR by maize is $1358.6 \mathrm{~mm}$ at Makhmur and the lowest at Chamchamal $588.1 \mathrm{~mm}$ (Table 8).

Table 8: Estimated CWR and IR for maize in the studied stations

\begin{tabular}{lccccccccccccc}
\hline \multirow{2}{*}{ Station } & \multicolumn{2}{c}{ Apr. } & \multicolumn{3}{c}{ May } & \multicolumn{2}{c}{ Jun. } & \multicolumn{3}{c}{ Jul. } & \multicolumn{2}{c}{ Aug. } & \multicolumn{2}{c}{ Total } \\
\cline { 2 - 14 } & CWR & IR & CWR & IR & CWR & IR & CWR & IR & CWR & IR & CWR & IR \\
\hline Akre & 33 & 0 & 115.2 & 99.5 & 221.8 & 221.6 & 245.8 & 245.8 & 138.6 & 138.5 & 754.4 & 705.4 \\
\hline
\end{tabular}


Water requirements for major crops in different agro-climatic zones of Iraqi Kurdistan using by CROPWAT 8.0

\begin{tabular}{lcccccccccccc} 
Chamchamal & 35.3 & 0 & 96.9 & 85.4 & 182.5 & 181.7 & 187.1 & 187.1 & 134 & 133.9 & 635.8 & 588.1 \\
\hline Duhok & 31.4 & 0 & 103.9 & 84.6 & 218.4 & 217.5 & 239.9 & 239.8 & 125.2 & 125.1 & 718.8 & 667 \\
\hline Erbil & 39.4 & 4 & 138.1 & 125.4 & 291.9 & 290.1 & 315.1 & 314.9 & 168.3 & 168.1 & 952.8 & 902.5 \\
\hline Makhmur & 52.6 & 15.4 & 192.5 & 184.5 & 431.9 & 431.4 & 478.5 & 478.5 & 248.9 & 248.8 & 1404.4 & 1358.6 \\
\hline Penjwen & 28.6 & 0 & 100.8 & 61.6 & 260.9 & 259.3 & 296.2 & 294 & 166.8 & 166.7 & 853.3 & 781.6 \\
\hline Soran & 30 & 0 & 112.2 & 89.5 & 267.7 & 266.5 & 300.9 & 300.3 & 161.3 & 160.6 & 872.1 & 816.9 \\
\hline Sulaymaniyah & 30.9 & 0 & 94.7 & 57.2 & 232.4 & 231.3 & 254.5 & 254.4 & 137.4 & 137.2 & 749.9 & 680.1 \\
\hline Zakho & 31.1 & 0 & 103.5 & 86.9 & 218.1 & 216.5 & 239.3 & 239.1 & 124.7 & 124.4 & 716.7 & 666.9
\end{tabular}

Potato

The highest CWR by potato is $704.1 \mathrm{~mm}$ at Makhmur and the lowest at Chamchamal 491.2mm; while, the highest IR by potato is $621.7 \mathrm{~mm}$ at Makhmur and the lowest at Chamchamal $401 \mathrm{~mm}$ (Table 9).

Table 9: Estimated CWR and IR for potato in the studied stations

\begin{tabular}{lcccccccccccc}
\hline \multirow{2}{*}{ Station } & \multicolumn{2}{c}{ Mar. } & \multicolumn{2}{c}{ Apr. } & \multicolumn{2}{c}{ May } & \multicolumn{2}{c}{ Jun. } & & Jul. & \multicolumn{2}{c}{ Total } \\
\cline { 2 - 6 } & CWR & IR & CWR & IR & CWR & IR & CWR & IR & CWR & IR & CWR & IR \\
\hline Akre & 21.4 & 0 & 76.1 & 23.9 & 195.2 & 179.5 & 206.2 & 206.1 & 65 & 65 & 563.9 & 474.5 \\
\hline Chamchamal & 27.4 & 8 & 81.7 & 23.5 & 165.5 & 153.8 & 167.9 & 167.1 & 48.7 & 48.6 & 491.2 & 401 \\
\hline Duhok & 23.3 & 4.1 & 72.3 & 22.6 & 174.1 & 155 & 200.4 & 199.5 & 63.9 & 63.8 & 534 & 445 \\
\hline Erbil & 28.6 & 2.1 & 92.2 & 51.1 & 232.4 & 219.6 & 266.7 & 264.9 & 84.2 & 84 & 704.1 & 621.7 \\
\hline Makhmur & 36.9 & 26.2 & 125.6 & 88.4 & 319.2 & 311.2 & 385.5 & 385.2 & 127.1 & 127.1 & 994.3 & 938.1 \\
\hline Penjwen & 19.7 & 0 & 67.7 & 2.2 & 167.6 & 125.1 & 236.3 & 234.7 & 78 & 77.2 & 569.3 & 439.2 \\
\hline Soran & 22.5 & 0 & 70.4 & 10.4 & 185.8 & 163.1 & 241.7 & 240.4 & 79.5 & 79.3 & 599.9 & 493.2 \\
\hline Sulaymaniyah & 23.5 & 0 & 70.9 & 8.9 & 156.5 & 119 & 211.2 & 210.1 & 67.4 & 67.3 & 529.5 & 405.3 \\
\hline Zakho & 22.9 & 0 & 71.7 & 26 & 173.5 & 157 & 200.1 & 198.5 & 63.8 & 63.7 & 532 & 445.2 \\
\hline
\end{tabular}

\section{Sunflower}

The highest CWR by sunflower is $704.1 \mathrm{~mm}$ at Makhmur and the lowest at Chamchamal 491.2mm; while, the highest IR by sunflower is $621.7 \mathrm{~mm}$ at Makhmur and the lowest at Chamchamal 401mm (Table 10).

Table 10: Estimated CWR and IR for sunflower in the studied stations

\begin{tabular}{lcccccccccccc}
\hline \multirow{2}{*}{ Station } & \multicolumn{2}{c}{ Mar. } & \multicolumn{2}{c}{ Apr. } & \multicolumn{2}{c}{ May } & \multicolumn{2}{c}{ Jun. } & & Jul. & \multicolumn{2}{c}{ Total } \\
\cline { 2 - 13 } & CWR & IR & CWR & IR & CWR & IR & CWR & IR & CWR & IR & CWR & IR \\
\hline Akre & 15 & 0 & 58.9 & 14.4 & 177.7 & 161.9 & 206.6 & 206.5 & 89.2 & 89.2 & 547.4 & 472 \\
\hline Chamchamal & 19.2 & 0.9 & 63.8 & 13.9 & 153.5 & 142 & 171.2 & 170.5 & 65.9 & 65.8 & 473.6 & 393.1 \\
\hline Duhok & 16.3 & 0 & 56.3 & 13.3 & 160.5 & 141.1 & 203 & 202.1 & 87 & 87 & 523.1 & 443.5 \\
\hline Erbil & 20 & 0 & 72.4 & 33.2 & 216.8 & 204.1 & 273.2 & 271.4 & 113.8 & 113.6 & 696.2 & 622.3 \\
\hline Makhmur & 25.9 & 15 & 99.9 & 62.7 & 304.5 & 296.5 & 402.3 & 401.8 & 169.7 & 169.7 & 1002.3 & 945.7 \\
\hline Penjwen & 13.8 & 0 & 53.7 & 0 & 158.8 & 116.3 & 245.5 & 243.9 & 105.3 & 103.7 & 577.1 & 463.9 \\
\hline Soran & 15.8 & 0 & 55.4 & 4.6 & 174.4 & 151.8 & 248.9 & 247.7 & 107.3 & 106.9 & 601.8 & 511 \\
\hline Sulaymaniyah & 16.4 & 0 & 55.1 & 3.1 & 144.5 & 107 & 214.2 & 213.1 & 91.5 & 91.4 & 521.7 & 414.6 \\
\hline Zakho & 16.1 & 0 & 55.8 & 14.7 & 159.9 & 143.2 & 202.6 & 201 & 86.9 & 86.7 & 521.3 & 445.6
\end{tabular}

Sweet Melon

The highest CWR by sweet melon is $936 \mathrm{~mm}$ at Makhmur and the lowest at Chamchamal 460.9mm; while, the highest IR by sweet melon is $879.5 \mathrm{~mm}$ at Makhmur and the lowest at Chamchamal $371.1 \mathrm{~mm}$ (Table 11).

Table 11: Estimated CWR and IR for sweet melon in the studied stations 
Water requirements for major crops in different agro-climatic zones of Iraqi Kurdistan using by CROPWAT 8.0

\begin{tabular}{lcccccccccccc}
\hline \multirow{2}{*}{ Station } & \multicolumn{2}{c}{ Mar. } & \multicolumn{2}{c}{ Apr. } & \multicolumn{2}{c}{ May } & \multicolumn{2}{c}{ Jun. } & \multicolumn{2}{c}{ Jul. } & \multicolumn{3}{c}{ Total } \\
\cline { 2 - 13 } & CWR & IR & CWR & IR & CWR & IR & CWR & IR & CWR & IR & CWR & IR \\
\hline Akre & 21.4 & 0 & 70.3 & 18.1 & 174.8 & 159.1 & 198.8 & 198.6 & 66.1 & 66.1 & 531.4 & 441.9 \\
\hline Chamchamal & 27.4 & 8 & 75.4 & 17.3 & 147.7 & 136.2 & 161.2 & 160.5 & 49.2 & 49.1 & 460.9 & 371.1 \\
\hline Duhok & 23.3 & 4.1 & 66.8 & 17.3 & 156.3 & 137 & 193.3 & 192.4 & 64.7 & 64.6 & 504.4 & 415.4 \\
\hline Erbil & 28.6 & 2.1 & 85 & 43.8 & 207.9 & 195.1 & 256.2 & 254.5 & 84.8 & 84.6 & 662.5 & 580.1 \\
\hline Makhmur & 36.9 & 26.2 & 115.4 & 78.2 & 286.1 & 278 & 370.2 & 369.7 & 127.4 & 127.4 & 936 & 879.5 \\
\hline Penjwen & 19.7 & 0 & 62.4 & 0 & 150 & 107.6 & 227 & 225.4 & 78.2 & 77.5 & 537.3 & 410.5 \\
\hline Soran & 22.5 & 0 & 64.8 & 6.4 & 166.8 & 144.1 & 233 & 231.7 & 80.1 & 79.9 & 567.2 & 462.1 \\
\hline Sulaymaniyah & 23.5 & 0 & 65.5 & 5.2 & 140.9 & 103.4 & 204.2 & 203.1 & 68.4 & 68.3 & 502.5 & 380 \\
\hline Zakho & 22.9 & 0 & 66.2 & 20.5 & 155.6 & 139.1 & 193 & 191.4 & 64.6 & 64.5 & 502.3 & 415.5 \\
\hline
\end{tabular}

\section{Tomato}

The highest CWR by tomato is $1229 \mathrm{~mm}$ at Makhmur and the lowest at Chamchamal $604.2 \mathrm{~mm}$; while, the highest IR by tomato is $1159.3 \mathrm{~mm}$ at Makhmur and the lowest at Chamchamal $491.6 \mathrm{~mm}$ (Table 12).

Table 12: Estimated CWR and IR for tomato in the studied stations

\begin{tabular}{lcccccccccccc}
\hline \multirow{2}{*}{ Station } & \multicolumn{2}{c}{ Mar. } & \multicolumn{2}{c}{ Apr. } & \multicolumn{2}{c}{ May } & \multicolumn{2}{c}{ Jun. } & \multicolumn{2}{c}{ Jul. } & \multicolumn{3}{c}{ Total } \\
\cline { 2 - 14 } & CWR & IR & CWR & IR & CWR & IR & CWR & IR & CWR & IR & CWR & IR \\
\hline Akre & 42.9 & 2.5 & 90.8 & 35.5 & 195.5 & 179.7 & 219.3 & 219.2 & 142.2 & 142.2 & 690.7 & 579.1 \\
\hline Chamchamal & 56.7 & 16.1 & 98.6 & 38.8 & 165.7 & 154.3 & 177.9 & 177.2 & 105.3 & 105.2 & 604.2 & 491.6 \\
\hline Duhok & 47.3 & 9 & 86.6 & 35.5 & 174.7 & 155.5 & 213.3 & 212.4 & 138.7 & 138.6 & 660.6 & 551 \\
\hline Erbil & 57.8 & 6 & 110.8 & 69.5 & 232.9 & 220.1 & 283 & 281.3 & 181.6 & 181.4 & 866.1 & 758.3 \\
\hline Makhmur & 74.4 & 50.5 & 151.5 & 114.3 & 320.8 & 312.7 & 408.9 & 408.5 & 273.4 & 273.3 & 1229 & 1159.3 \\
\hline Penjwen & 39.5 & 0 & 81.6 & 5.5 & 168.2 & 125.6 & 250.9 & 249.3 & 168.7 & 166.9 & 708.9 & 547.3 \\
\hline Soran & 45.4 & 0 & 84.5 & 13.8 & 186.7 & 164.1 & 257.1 & 255.9 & 172.3 & 171.9 & 746 & 605.7 \\
\hline Sulaymaniyah & 47.9 & 0 & 85.2 & 12.1 & 157.5 & 120 & 225.2 & 224 & 146.5 & 146.4 & 662.3 & 502.5 \\
\hline Zakho & 46.6 & 0.1 & 85.9 & 37.7 & 174.1 & 157.5 & 213 & 211.3 & 138.5 & 138.4 & 658.1 & 545 \\
\hline
\end{tabular}

\section{Wheat}

The highest CWR by wheat is $832 \mathrm{~mm}$ at Makhmur and the lowest at Penjwen $440.4 \mathrm{~mm}$; while, the highest IR by wheat is $625.2 \mathrm{~mm}$ at Makhmur and the lowest at Penjwen 119mm (Table 13).

Table 13: Estimated CWR and IR for wheat in the studied stations

\begin{tabular}{|c|c|c|c|c|c|c|c|c|c|c|c|c|c|c|c|c|c|c|}
\hline \multirow{2}{*}{ Station } & \multicolumn{2}{|c|}{ Nov. } & \multicolumn{2}{|c|}{ Des. } & \multicolumn{2}{|c|}{ Jan. } & \multicolumn{2}{|c|}{ Feb. } & \multicolumn{2}{|c|}{ Mar. } & \multicolumn{2}{|c|}{ Apr. } & \multicolumn{2}{|c|}{ May } & \multicolumn{2}{|c|}{ Jun. } & \multicolumn{2}{|c|}{ Total } \\
\hline & CWR & IR & CWR & IR & CWR & IR & CWR & IR & CWR & IR & CWR & IR & CWR & IR & CWR & IR & CWR & IR \\
\hline Akre & 16.4 & 0 & 24 & 0 & 25.5 & 0 & 41.5 & 0 & 78.6 & 32.6 & 120.9 & 65.5 & 138.4 & 122.7 & 32.1 & 32 & 477.4 & 252.8 \\
\hline Chamchamal & 20.9 & 5 & 31.5 & 2.4 & 41.1 & 0 & 67.3 & 0.2 & 112.2 & 71.7 & 139.7 & 79.9 & 121 & 109.5 & 26.1 & 25.4 & 559.8 & 294.1 \\
\hline Duhok & 15.7 & 0 & 23.5 & 0 & 28.8 & 0 & 45.3 & 0 & 88.3 & 46.5 & 117.1 & 65.9 & 123.7 & 104.4 & 30.6 & 29.7 & 473 & 246.5 \\
\hline Erbil & 19.5 & 0.7 & 29.5 & 0 & 36 & 0 & 57.9 & 2 & 113 & 57.6 & 153.5 & 112.3 & 166 & 153.3 & 40 & 38.5 & 615.4 & 364.4 \\
\hline Makhmur & 26.8 & 16 & 38.3 & 13 & 47.7 & 6.2 & 74.6 & 15.1 & 150.5 & 126.4 & 212.2 & 175.1 & 226.3 & 218.2 & 55.6 & 55.2 & 832 & 625.2 \\
\hline Penjwen & 14.5 & 0 & 24.1 & 0 & 23.1 & 0 & 36.5 & 0 & 78.2 & 0 & 113.3 & 12.3 & 117.3 & 74.9 & 33.4 & 31.8 & 440.4 & 119 \\
\hline Soran & 15 & 0 & 20.1 & 0 & 23.1 & 0 & 36.6 & 0 & 87.9 & 9.4 & 115.9 & 29.9 & 131.1 & 108.4 & 35.2 & 34 & 464.9 & 181.7 \\
\hline Sulaymaniyah & 14.9 & 0 & 23.7 & 0 & 27.7 & 0 & 46.7 & 0 & 90.2 & 14.9 & 116.5 & 35.5 & 111.9 & 74.4 & 31.6 & 30.5 & 463.2 & 155.3 \\
\hline Zakho & 15.2 & 0 & 22.5 & 0 & 27.9 & 0 & 44.1 & 0 & 87.1 & 25.6 & 116.1 & 67.8 & 123.1 & 106.6 & 30.5 & 28.9 & 466.5 & 228.9 \\
\hline
\end{tabular}


Water requirements for major crops in different agro-climatic zones of Iraqi Kurdistan using by CROPWAT 8.0

\section{CONCLUSION}

Results of the study explain that the evapotranspiration $\left(\mathrm{ET}_{\mathrm{o}}\right)$ was highest in July $(12 \mathrm{~mm} /$ day $)$ and lowest in January $(0.9 \mathrm{~mm} /$ day $)$. On the other hand, estimated CWR and IR show that the use of water and irrigation projects should be done in Kurdistan region, especially in Karkuk zone, with modern methods to increase efficiency and save water resources.

\section{RECOMMENDATIONS}

It is recommended that prepared a comprehensive plan to estimation CWR for all zones in Iraqi Kurdistan region. Such a plan can be used as a basis for agricultural projects. However, estimated CWR and IR must be confirmed by practical tests.

\section{Journal Papers:}

\section{REFERENCES}

[1] A. Hadi and M. Mehdi, Water requirement of tomato using software by CROPWAT version 8i, Zabol University water engineering journal, 2010. (In Persian).

[2] Adeniran K.A. et al., Water requirements of some selected crops in Kampe dam irrigation project, Australian Journal for agricultural engineering, AJAE 1(4), 2010, 119-125.

[3] J. Birkenshaw and J. Bailey, Irrigation Best Practice - A Guide for Vegetable Growers, ADAS Horticulture with funding from the Department for Environment, Food and Rural Affairs, 2003.

[4] J. Tekwa and E. K. Bwade, Estimation of Irrigation Water Requirement of Maize (Zea-mays) using Pan Evaporation Method in Maiduguri, Northeastern Nigeria. Agricultural Engineering International: the CIGR Journal, Vol-13, No.1, 2011, Manuscript No.1552.

[5]W. J. Sacks, D. Deryng, J. A. Foley and N. Ramankutty, Crop planting dates: an analysis of global patterns, Global Ecology and Biogeography, 19, 2010, 607-620.

\section{Books:}

[6] A. M. Michael, Irrigation Theory and Practice. Vikas Publishing House, New Delhi, India, 1999.

\section{Chapters in Books:}

[7] Chapter 11, "Sprinkler Irrigation” Section 15, Natural Resources Conservation Service National Engineering Handbook, 1990.

\section{Proceedings Papers:}

[8] A. Muhammad and M. Abdul, Water requirements of major crops for different agro-climatic zones of Balochistan, The World Conservation Union (IUCN), 2008

[9] Doorenbus J. and Pruitt W.O, Guide lines for predicting Crop water requirements, FAO Irrigation and Drainage Paper No. 24.1977.

[10] FAO, Example of the Use of Cropwat 8.0, The Rajolibanda Diversion Scheme in Andhra Pradesh, India, 2008.

[11] FAO, Irrigation water requirements, In: Irrigation Potential in Africa: A Basin Approach, FAO Corporate Document Repository, Rome. Chapter 5, 2005, http://www.fao.org/docrep/W 4347E /w4347e00.htm

[12] Hess, T.M. and T.-M. Stein, DailyET - A simple 'calculator' for estimating daily reference crop evapotranspiration (ETo), 2005, http://www.irrisoft.org.

[13] M. A. Tara, Country pasture/forage resource profiles Iraq, Research and Extension, FAO, 2011.

[14] M. Ashraf and A. Majeed, Water requirements of major crops for different agro-climatic zones of Balochistan, The World Conservation Union (IUCN) Pakistan, Water Program, 2006.

[15] M. Smith, CROPWAT-A Computer Program for Irrigation Planning and Management, FAO Irrigation and Drainage Paper No. 46, 1992.

[16] Ministry of Planning Kurdistan Regional Government, Agricultural areas in the governorates of Kurdistan Region, KRSO report, 2012. 
Water requirements for major crops in different agro-climatic zones of Iraqi Kurdistan using by CROPWAT 8.0

[17] MOAKR, The status of agriculture production: an overview, report to Kurdistan Strategic plan for agriculture, Erbil, 2008.

[18] P. Steduto, et al., Crop yield response to water, FAO Irrigation and Drainage Paper No. 66, 2012.

[19] Richard G. et al., Crop evapotranspiration (Guidelines for Computing Crop Water Requirements), FAO Irrigation and Drainage Paper No. 56, 1998.

\section{Appendix}

Table App. 1: Main features of the crops studied

\begin{tabular}{llccccc}
\multirow{2}{*}{$\begin{array}{l}\text { Brop } \\
\text { Alfalfa }\end{array}$} & \multirow{2}{*}{ Botanical Name } & Planting time & Harvesting time & \multicolumn{2}{c}{ Kc, Crop coefficient } \\
\cline { 5 - 7 } & Medicago sativa L. & Mar-Apr & May-Jun & 0.4 & Mid & End \\
\hline Barley & Hordeum vulgare L. & Mid Nov & May & 0.6 & 1.2 & 1.15 \\
\hline Beans & Phaseolus coccineus L. & Oct-Nov & May & 0.4 & 1.15 & 0.35 \\
\hline Vegetable(Cabbage) & Brassica oleracea & Agu & Mar & 0.7 & 1.05 & 0.95 \\
\hline Grape & Vitis vinifera & Feb & Aug-Sep & 0.3 & 1.05 & 0.5 \\
\hline Maize (Grain) & Zea mays L. & Mar-Apr & Jul & 0.3 & 1.2 & 0.4 \\
\hline Potato (spring) & Solanum Tuberosum & Feb-Mar & Jun & 0.5 & 1.15 & 0.75 \\
\hline Sunflower & Helian Thusannuus L. & Mar & Jul & 0.35 & 1.1 & 0.25 \\
\hline Sweet Melon & Cucumis melo L. & Mar & Jun-Jul & 0.5 & 1.05 & 0.75 \\
\hline Tomato & Solanum lycopersicum & Mar-Apr & Jul-Oct & 0.6 & 1.15 & 0.8 \\
\hline Wheat & Triticum aestivum & Mid Nov & Jun & 0.6 & 1.15 & 0.32 \\
\hline
\end{tabular}

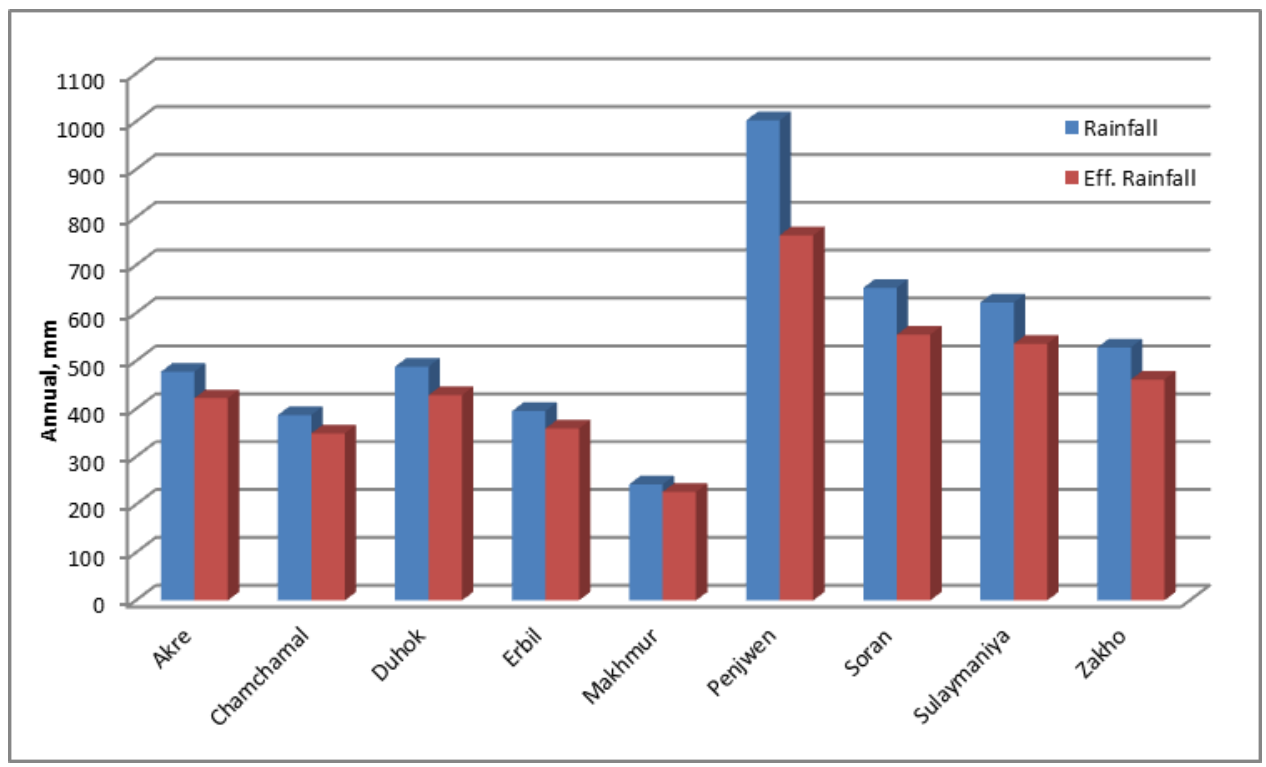

Figure App.1: Annual rainfall and effective rainfall by USDA S.C. Method for studied stations. 